\title{
APLICAÇÃO DAS FERRAMENTAS DA QUALIDADE PARA MELHORIA DO PROCESSO PRODUTIVO DE UMA INDÚSTRIA DE REFRIGERANTES
}

Amanda Letícia Remondini, Bruna de Oliveira Marcondes, Gleice Kelli de Alcântara Anastácio, Pedro Paulo Liberato Gomes, Andréia de Menezes Olivo

Universidade do Oeste Paulista - UNOESTE, Faculdade de Engenharia de Produção, Presidente Prudente, SP. E-mail: gleice kelli10@hotmail.com.

\section{RESUMO}

O presente artigo aborda como tema de estudo a engenharia da qualidade, que é a área da Engenharia de Produção destinada ao planejamento, projeto e controle de sistemas de gestão da qualidade baseados no gerenciamento por processos, na abordagem de fatos para a tomada de decisão e na utilização das ferramentas da qualidade. Nesta área, o foco do artigo está direcionado à aplicação das ferramentas da qualidade em uma das linhas de produção de uma indústria de refrigerantes. O objetivo do estudo é mensurar as perdas geradas no processo de fabricação e propor meios para controlar a sua incidência, a fim de reduzir custos e aumentar a produtividade. A metodologia foi baseada em uma abordagem qualitativa e quantitativa, na aplicação do estudo de caso, e na pesquisa bibliográfica e informações fornecidas pela empresa como base de dados. Como resultados, as ferramentas da qualidade evidenciaram que o problema de baixo nível de líquido é o mais representativo e que suas principais causas se referem à inadequação da temperatura de envase e da posição do dispositivo nivelador de líquido, para as quais foram elaborados planos de ação que visam gerar melhorias no processo produtivo.

Palavras-chave: refugo; reprocesso; controle da qualidade; ferramentas da qualidade; aumento da produtividade.

\section{APPLICATION OF QUALITY TOOLS FOR IMPROVING THE PRODUCTION PROCESS OF A REFRIGERANT INDUSTRY}

\begin{abstract}
The present article deals with quality engineering, which is an area of Production Engineering for planning, design and control of quality management systems based on process management, in the approach to facts for decision making and the use of quality tools. In this area, the focus of the article is directed to the application of quality tools in one of the production lines of a refrigerant industry. The objective of the study is to measure the losses generated in the manufacturing process and propose means to control their incidence, in order to reduce costs and increase productivity. The methodology was based on a qualitative and quantitative approach, in the application of the case study, and in the bibliographic research and information provided by the company as a database. As results, the quality tools showed that the problem of low liquid level is the most representative and that its main causes refer to the inadequacy of the packaging temperature and the position of the liquid leveling device, for which action plans have been developed that aim generate improvements in the production process.
\end{abstract}

Keywords: rejects; reprocessing; quality control; quality tools; productivity increase. 


\section{INTRODUÇÃO}

O artigo aborda como tema de estudo a engenharia da qualidade, que para a Associação Brasileira de Engenharia de Produção (ABEPRO, 2008), trata-se do "planejamento, projeto e controle de sistemas de gestão da qualidade que considerem o gerenciamento por processos, a abordagem factual para a tomada de decisão e a utilização de ferramentas da qualidade."

De acordo com Lobo (2010), a qualidade é um conjunto de características de um produto ou serviço que lhe promovem aptidão para satisfazer as necessidades explícitas ou implícitas do consumidor. Sendo assim, a qualidade pode ser definida como a conformidade com os requisitos ou simplesmente como a satisfação do consumidor.

A qualidade é um conceito que evolui constantemente, dessa forma, é necessário que seus requisitos sejam revisados periodicamente para que sejam atualizados de acordo com as novas exigências e expectativas do mercado. Dessa forma, a qualidade é um fator altamente competitivo e é um dos principais objetivos de desempenho que uma empresa deve almejar, pois a oferta de um produto ou serviço de qualidade resulta em credibilidade no mercado, que por consequência gera uma maior rentabilidade para a empresa.

O controle de qualidade, por sua vez, pode ser definido como um conjunto de técnicas e atividades operacionais que possuem a finalidade de satisfazer os requisitos de qualidade preestabelecidos. A partir do monitoramento das etapas do processo produtivo e da identificação de deficiências, busca-se minimizar as falhas e defeitos, aumentar a produtividade e consequentemente reduzir os custos de produção. (LOBO, 2010)

Este artigo consiste no estudo de uma empresa do ramo de fabricação de refrigerantes, cujos problemas relacionados à qualidade são encontrados na linha de produção destinada ao envase de refrigerantes em garrafas pet de $250 \mathrm{ml}$ a $2000 \mathrm{ml}$, e são representados por: refugos de polietileno tereftalato (pet), rolhas, rótulos, pacotes; ou ainda problemas de baixo nível de líquido, havendo a necessidade de reprocesso ou descarte da unidade.

Os problemas identificados já são de conhecimento da empresa, porém devido à alta frequência são considerados normais e ignorados. Os mesmos ocasionam desperdício de matériaprima, paradas de linha não programadas e uma consequente queda de produtividade, acarretando custos indesejados e atrasos no cumprimento da programação de produção. Diante deste cenário, este artigo tem como objetivo principal mensurar as referidas perdas e, através da aplicação das ferramentas da qualidade, encontrar meios para controlar estatisticamente a incidência das perdas de maior representatividade e assim aumentar a produtividade da empresa.

\section{PROCESSO DE FABRICAÇÃO DE REFRIGERANTES}

A partir de um acompanhamento do processo de fabricação de refrigerantes da empresa em questão, observou-se que o mesmo é bastante simples, onde a desagregação ocorre numa única etapa, a qual define o sabor, a cor e o aroma do produto que está sendo preparado.

Após o recebimento do açúcar, o mesmo é dissolvido em água e essa mistura é aquecida a uma temperatura de $85^{\circ} \mathrm{C}$. A substância obtida é chamada de xarope simples, que posteriormente é filtrada com alguns materiais auxiliares. Em seguida, são adicionados conservantes, aromatizantes e corantes para gerar o xarope composto. Este xarope, por sua vez, necessita de um período de descanso e resfriamento antes de ser complementado com água carbonatada.

Depois da desagregação, o xarope composto é levado à injetora por tubulações que mantêm o líquido resfriado através de serpentinas envoltas por amônia. As garrafas pet utilizadas no envase podem ser sopradas no momento da produção ou estocadas previamente, sendo adicionadas à linha de produção manualmente minutos antes do processo. Na sopradora, ocorrem dois tipos de refugos, os decorrentes de defeitos presentes na preforma enviada pelo fornecedor, ou aqueles gerados pela própria máquina, resultando em garrafas deformadas. 
Uma vez soprada e aprovada, a garrafa continua no processo, sendo encaminhada para a etapa de envase, onde o líquido preparado anteriormente é colocado na garrafa através da injetora. Neste maquinário, ocorrem refugos de garrafas amassadas ou deformadas que não foram vistas na inspeção da sopradora ou foram corrompidas no trajeto da sopradora à injetora. Na própria injetora, um componente fecha as rolhas plásticas na garrafa, onde pode ocorrer outro tipo de refugo, caracterizado por rolhas não conformes com os padrões definidos pela empresa, como irregularidades no logotipo ou no lacre, por exemplo. Após o envase, a garrafa passa por um dispositivo nivelador de líquido, que visa retirar excessos para a padronização do volume. Ainda na etapa de envase, pode haver a necessidade de reprocesso ou descarte da unidade devido a um baixo nível de líquido na garrafa.

Depois de revisado em questão de nível, rolha e garrafa, o produto vai para a rotuladora, onde são gerados refugos decorrentes de rótulos colados inadequadamente. Em seguida, as garrafas são agrupadas em um determinado número de unidades, de acordo com o seu volume, e são encaminhadas para o empacotamento, onde são envoltas por um plástico e então passam por uma máquina de aquecimento, de modo a conformar o plástico às garrafas. Nesta etapa, ocorrem refugos de pacotes quando o plástico se rompe ou não se fixa adequadamente. Por fim, as garrafas empacotadas são paletizadas e encaminhadas para a estocagem.

É importante destacar que o produto é reprocessado nas etapas que ocasionaram refugos e geraram não conformidades desde que a correção seja possível, já os refugos que foram efetivamente perdidos são descartados.

\section{METODOLOGIA}

O artigo foi desenvolvido sob uma perspectiva qualitativa e quantitativa, permitindo assim uma maior abrangência à pesquisa. $O$ estudo de caso foi utilizado com o intuito de investigar e compreender o contexto real da linha de produção abordada. A coleta de dados foi baseada na pesquisa bibliográfica, como suporte teórico, além de registros da própria empresa. E por fim, o universo de estudo se limitou aos refugos e reprocessos existentes em uma das linhas de produção da indústria em questão, mais especificamente uma linha destinada ao envase de refrigerantes em garrafas pet.

O estudo dos problemas encontrados na referida linha de produção foi baseado na aplicação de algumas ferramentas da qualidade, através das quais foi possível compreender o processo e desenvolver planos de ação afim de controlar os problemas constatados, visto que proporcionam uma síntese objetiva dos dados a partir de uma exposição gráfica dos mesmos. As ferramentas utilizadas foram a folha de verificação, a estratificação, o diagrama de Pareto, o diagrama de causa e efeito, o gráfico de dispersão e a carta controle, além da técnica 5W2H (what - o que; why - por que; who - quem; where - onde; when - quando; how - como; how much quanto), que de acordo com Seleme e Stadler (2012), possui o objetivo de esclarecer o problema analisado e gerar ideias para solucioná-lo.

\section{REVISÃO DE LITERATURA}

\section{FERRAMENTAS DA QUALIDADE}

Segundo Seleme e Stadler (2012), as folhas de verificação são documentos que registram as anotações direcionadas ao problema em estudo. Barros e Bonafini (2014) afirmam que esse formulário pode ser desenvolvido para coletar dados suficientes relacionados às questões necessárias para gerar respostas, sendo possível observar a concentração de falhas e aplicar outras ferramentas da qualidade para descobrir as causas dos problemas e tratá-las. A estratificação, por sua vez, é uma ferramenta que permite a separação de grupos em subgrupos 
específicos, que por consequência possibilita analisar um conjunto de problemas a fim de encontrar a causa principal destes. (SELEME; STADLER, 2012)

De acordo com Seleme e Stadler (2012), o diagrama de Pareto é uma ferramenta que se baseia em uma relação de $20 / 80$, que significa que $80 \%$ dos problemas são provenientes de $20 \%$ das causas. Com isso, é possível dividir os problemas em vitais e triviais, ou seja, a menor quantidade de causas é responsável pelos maiores defeitos. A partir disso, o gráfico possibilita a identificação do problema que deve ser trabalhado prioritariamente e quais devem ser os próximos a serem corrigidos ou controlados.

De acordo com Barros e Bonafini (2014), o diagrama de causa e efeito ou diagrama de Ishikawa é usado para identificar a relação entre a causa e o efeito de um processo analisando os materiais, mão de obra, método, máquina, medição e meio ambiente (6 M's). Segundo Seleme e Standler (2012, p. 91), "essa representação gráfica permite estabelecer [...] quais são as causas [...] que fazem com que o efeito ocorra. É um diagrama que pretende mostrar a relação entre uma característica da qualidade e seus diversos fatores determinantes."

Seleme e Stadler (2012) relatam que o diagrama de dispersão ou correlação consiste em um gráfico que a partir da dispersão dos pontos permite analisar a interdependência entre duas variáveis, indicando inclusive os parâmetros de variação que definem o padrão de qualidade. Por fim, a carta controle é uma ferramenta da qualidade que permite observar a variação de dados referentes à medição presente em um processo, mostrando se ela se encontra dentro do limite de tolerância ou se apresenta desvios que necessitam de investigação, o que torna possível a busca pela padronização dos parâmetros observados. (BARROS; BONAFINI, 2014)

\section{RESULTADOS}

O período de observação corresponde aos dias de operação da linha de produção em estudo durante o mês de março de 2017. Conforme a metodologia apresentada, a coleta de dados foi baseada no preenchimento da folha de verificação. Neste documento, foram quantificados os refugos de garrafas deformadas, preformas inadequadas, rolhas, rótulos e pacotes, além dos reprocessos ou descartes necessários devido à problemas com baixo nível de líquido no envase. Estes problemas, por sua vez, foram devidamente categorizados a partir da estratificação.

Conforme a Figura 1, através da aplicação do diagrama de Pareto, constatou-se que os refugos de rolha e baixo nível de líquido são os mais representativos, visto que correspondem, respectivamente, a $49,22 \%$ e $33,89 \%$ dos refugos considerados. Ou seja, $83,11 \%$ dos refugos desta linha de produção se referem a essas duas classificações, o que refina o universo de estudo e proporciona um melhor enfoque para a aplicação das demais ferramentas da qualidade. 
Figura 1 . Diagrama de Pareto: Identificação dos problemas mais representativos

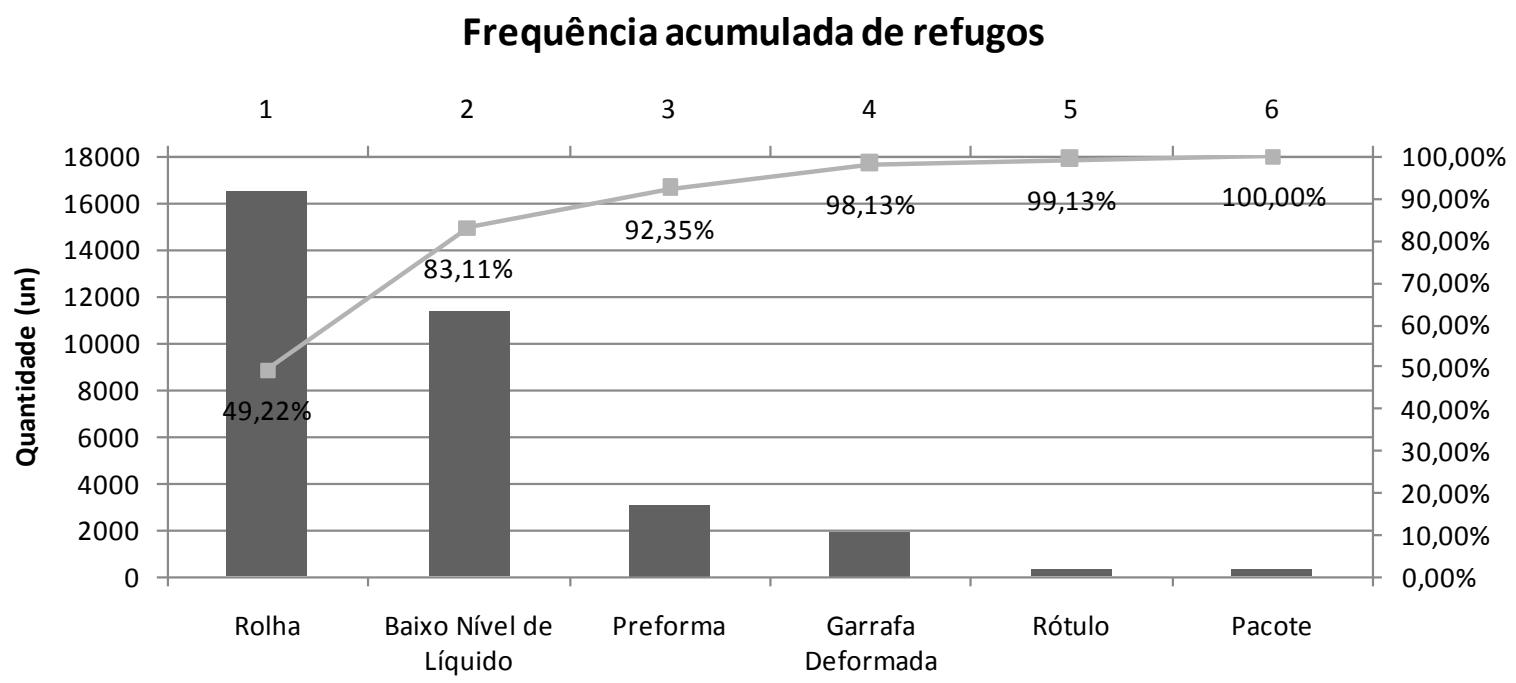

Fonte: Elaborado pelos próprios autores.

Considerando o refugo de baixo nível de líquido como o principal, conforme a justificativa apresentada na seção de discussão, o diagrama de causa e efeito foi aplicado para destacar as possíveis causas primárias deste problema, cuja classificação se deu a partir dos 6 M's, conforme apresentado na Figura 2.

Figura 2. Diagrama de causa e efeito: Classificação 6M's

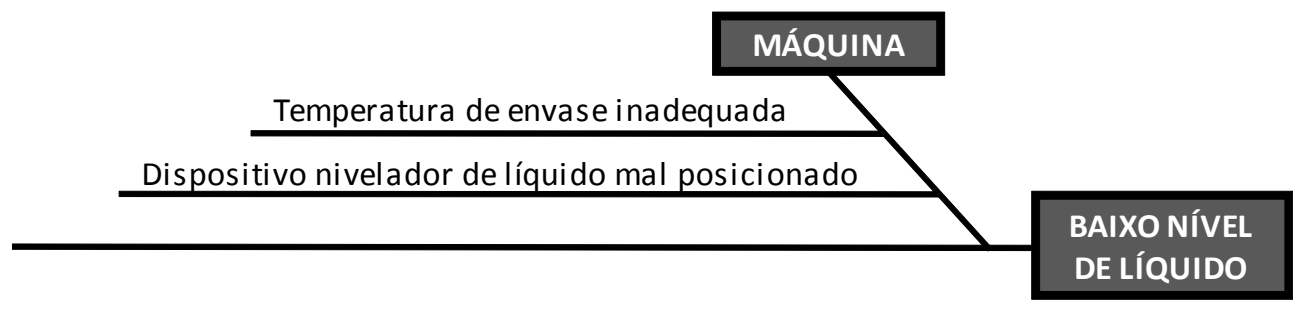

Fonte: Elaborado pelos próprios autores.

Foram propostos planos de ação através da técnica $5 \mathrm{~W} 2 \mathrm{H}$ para intervir nas possíveis causas elencadas no diagrama de Ishikawa, referentes ao problema de refugo ou reprocesso a partir da etapa de envase devido ao baixo nível de refrigerante nas garrafas.

O diagrama de dispersão ou correlação foi aplicado com o intuito de verificar se realmente há relação entre a temperatura de envase e o volume envasado, ou seja, se a temperatura do líquido exerce alguma influência sobre o volume injetado na garrafa. Observando a Figura 3, é possível afirmar que há um considerável grau de influência da temperatura do líquido sobre o volume de envase, pois a coeficiente de correlação obtido equivale a 71,5\%. 
Figura 3. Diagrama de dispersão: Relação entre temperatura e volume

Relação entre temperatura de envase e volume envasado

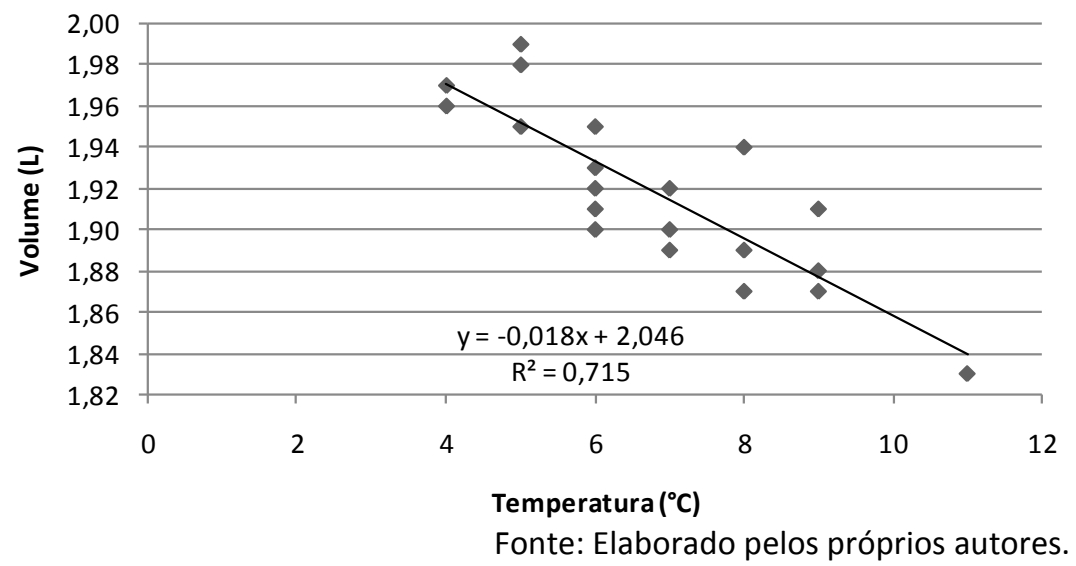

A carta controle, apresentada na Figura 4, foi aplicada neste estudo para analisar a variabilidade da temperatura de envase a partir de conjuntos de duas amostras retirados em dias aleatórios do período observado, visto que, como constatado no diagrama de dispersão, esta variável exerce uma significativa influência sobre o problema principal.

Figura 4. Carta controle: Variabilidade da temperatura de envase
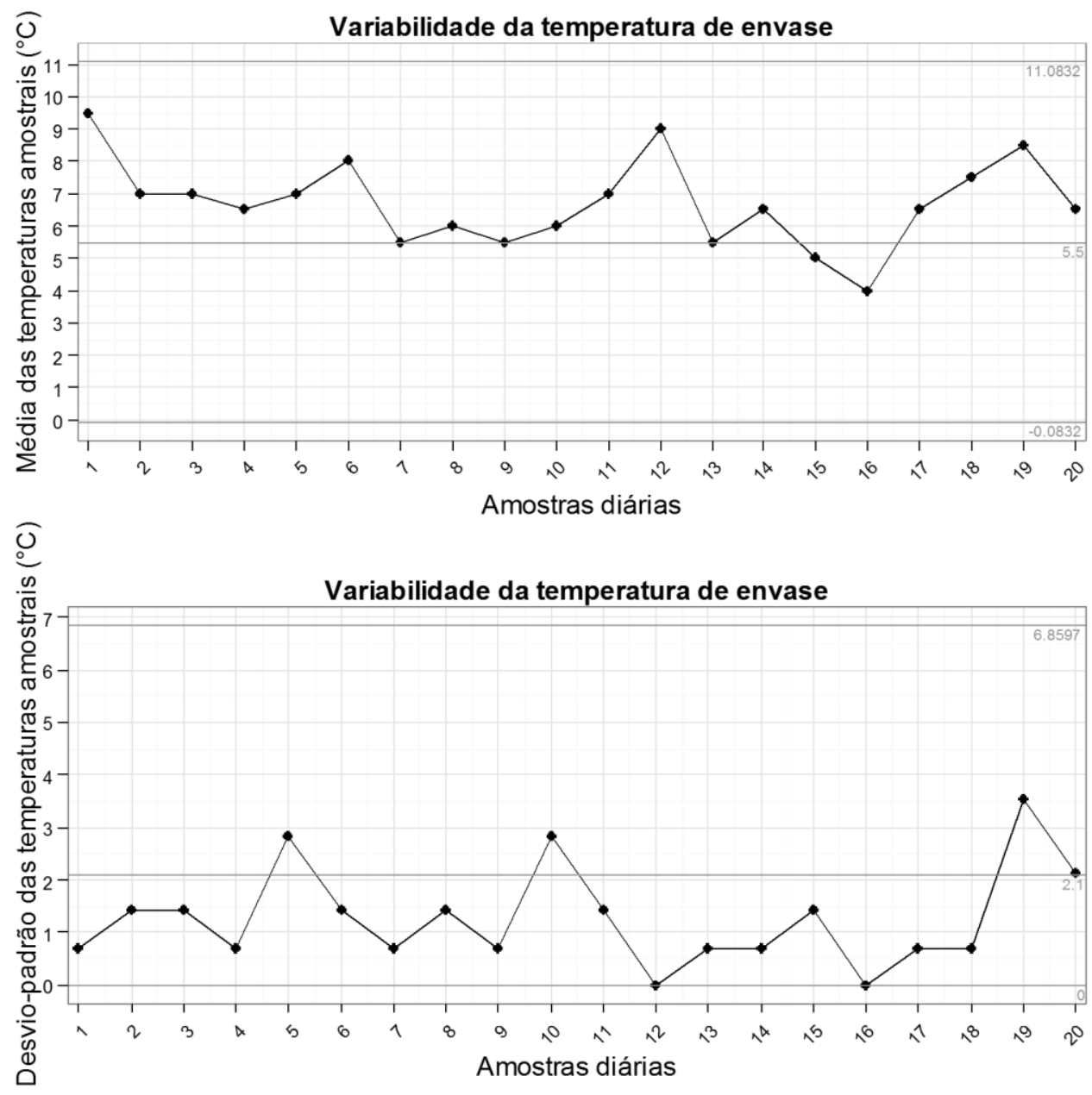

Fonte: Elaborado pelos próprios autores. 


\section{DISCUSSÃO}

É importante destacar que o refugo de rolha é consequente do refugo por baixo nível de líquido na garrafa e que no diagrama de Pareto não foram consideradas as rolhas que já são recebidas do fornecedor com não conformidades. Sendo assim, observa-se que a alta taxa de refugos de rolha neste processo se deve ao fato de que a maioria das garrafas com baixo nível de líquido é reprocessada, ou seja, elas retornam à linha de produção para completar o enchimento e outra rolha é utilizada. Diante disso, as ferramentas da qualidade aplicadas nas etapas seguintes dão prioridade ao controle do refugo/reprocesso por baixo nível de líquido, visto que se o mesmo for alcançado, certamente o índice de refugos de rolha também será controlado.

Em relação aos planos de ação propostos para as causas do problema principal, a temperatura de envase foi considerada inadequada visto que atualmente ocorrem frequentes variações no volume de líquido em decorrência da perda de gás carbônico (CO2), sendo assim, é necessário controlá-la entre $4^{\circ} \mathrm{C}$ e $7^{\circ} \mathrm{C}$, que é o intervalo de temperatura indicado para manter a qualidade do refrigerante e realizar o envase na quantidade correta. Para tanto, o departamento de manutenção com o auxílio do controle de qualidade deve promover imediatamente a regulagem dos resfriadores da linha de produção e desenvolver um constante acompanhamento.

Já a afirmação de que o dispositivo nivelador de líquido está mal posicionado se deve ao fato de que a posição atual do mesmo faz com que o nível de líquido gaseificado seja ajustado antes de sua estabilização, visto que ao injetar o refrigerante nas garrafas, as moléculas permanecem agitadas por um tempo, e o ajuste do volume durante o período instável incide no excesso de retirada de líquido. Diante disso, é necessário que o departamento de manutenção em conjunto com o de produção providencie de forma imediata a realocação do dispositivo nivelador em um ponto da linha de produção cujo tempo de transporte da etapa de envase até o mesmo seja suficiente para a estabilização do refrigerante. Além disso, este dispositivo deve ser regulado frequentemente, de modo a exercer exatamente o esforço necessário para padronizar o volume.

As variáveis analisadas no gráfico de dispersão apresentam uma correlação negativa, ou seja, quanto maior a temperatura de envase, menor é o volume de líquido injetado. Sendo assim, se o plano de ação referente ao controle da temperatura de envase for executado com êxito, é altamente provável que o índice de reprocesso ou refugo por baixo nível de líquido seja reduzido.

Conforme a carta controle apresentada, embora haja uma alta instabilidade nas temperaturas medidas, os valores não ultrapassam os limites de tolerância, que para a média amostral são de aproximadamente $-0,08^{\circ} \mathrm{C}$ e $11,08^{\circ} \mathrm{C}$, valores estes que não se distanciam muito dos especificados pela própria empresa, que são de $3^{\circ} \mathrm{C}$ e $14^{\circ} \mathrm{C}$. A fim de realizar uma melhor análise, a média e o desvio-padrão entre os limites de tolerância ideais propostos no plano de ação $\left(4^{\circ} \mathrm{C}\right.$ e $\left.7^{\circ} \mathrm{C}\right)$ foram fixados nos gráficos, correspondendo respectivamente a $5,5^{\circ} \mathrm{C}$ e $2,1^{\circ} \mathrm{C}$. Com essas informações, é possível observar que a maioria dos conjuntos amostrais apresenta um desvio-padrão dentro do intervalo indicado, a média, no entanto, varia de maneira mais significativa, apresentando poucas amostras com a média de temperatura desejada.

Portanto, apesar do sistema estar aparentemente sob controle neste aspecto, é indicado que as especificações de temperatura mínima e máxima sejam ajustadas e que a variabilidade seja menor, a fim de proporcionar melhores resultados na linha de produção a partir da minimização dos reprocessos e refugos decorrentes de baixo nível de líquido, pois as especificações atuais não têm sido satisfatórias. Dessa forma, a validação do plano de ação proposto se mostra extremamente importante para promover essas melhorias, a partir da qual os limites de tolerância especificados passariam a ser de $4^{\circ} \mathrm{C}$ e $7^{\circ} \mathrm{C}$. 


\section{CONSIDERAÇÕES FINAIS}

No estudo desenvolvido, foi possível observar a utilidade e a importância das ferramentas da qualidade como forma racional e metódica de identificar os principais problemas de uma linha de produção e suas respectivas causas, além disso, essas ferramentas evidenciam as informações mais relevantes dos dados analisados e assim possibilitam a definição de planos de ação que visam controlar as não conformidades e promover melhorias no processo produtivo.

\section{REFERÊNCIAS}

ASSOCIAÇÃO BRASILEIRA DE ENGENHARIA DE PRODUÇÃO (ABEPRO). Áreas e Sub-áreas de Engenharia de Produção. Rio de Janeiro, 2008. Disponível em:

<http://www.abepro.org.br/interna.asp?p=399\&m=424\&ss=1\&c=362>. Acesso em: 16 mai. 2017.

BARROS, Elsimar; BONAFINI, Fernanda. Ferramentas da qualidade. São Paulo: Pearson Education do Brasil, 2014. Disponível em:

<http://unoeste.bv3.digitalpages.com.br/users/publications/9788543009940/pages/-12>. Acesso em: 16 mai. 2017.

LOBO, Renato Nogueirol. Gestão da Qualidade. 1. ed. São Paulo: Érica, 2010.

SELEME, Robson; STADLER, Humberto. Controle da qualidade: as ferramentas essenciais. Curitiba: InterSaberes, 2012. 